\title{
Do additional cores from cancer-suspicious lesions on transrectal ultrasound improve prostate cancer detection including index tumors over 12-core systematic biopsy?
}

This article was published in the following Dove Press journal:

Cancer Management and Research

Jung Ki Jo'

Sung Kyu Hong ${ }^{2}$

Seok-Soo Byun ${ }^{2}$

Sang Eun Lee ${ }^{2}$

Seong Jin Jeong ${ }^{2}$

'Department of Urology, Hanyang University Seoul Hospital, Seoul,

Korea; ${ }^{2}$ Department of Urology, Seoul National University Bundang Hospital, Seongnam, Korea
Correspondence: Seong Jin Jeong

Department of Urology, Seoul National University Bundang Hospital,

82, Gumi-ro, 173 Beon-gil, 463-707 Bundang-gu, Seongnam-si, Gyeonggi-do, Korea

$\mathrm{Tel}+82317877350$

Fax +82 31 7874057

Email urojsj@snubh.org
Objective: The efficacy of additional cores from cancer-suspicious lesions by transrectal ultrasound (TRUS) for the detection of prostate cancer (PCa) was assessed.

Materials and methods: Data were collected from 4144 men who underwent systematic 12-core biopsy with no cancer-suspicious lesions (Group A: 3256 cases) or 13- or more-core (systematic 12 core + additional cores) biopsy with cancer-suspicious lesions (Group B: 888 cases) on TRUS-guided biopsy. The effect of additional biopsy cores on the cancer detection rate was investigated.

Results: PCa was detected in 1006 (30.9\%) cases in Group A and 485 (54.6\%) cases in Group B $(p<0.001)$. In 370/485 (76.3\%) patients in Group B, cancer was detected from the additional cores from TRUS suspicious lesions. Logistic regression analysis showed that the number of biopsy cores was the most significant factor for cancer detection (hazards ratio: 2.6 [2.2213.043], $p<0.001]$. Additional core biopsies did not increase the detection rate of index tumors $(p<0.001)$. However, the Gleason score of index tumors was higher than that of systematic cores $(p<0.001)$. Kaplan-Meier analysis showed no significant differences in survival according to biopsy number and location of index tumors (log-rank test: $p=0.583, p=0.165$, respectively). Conclusion: Although additional core biopsies can increase the cancer detection rate, they do not increase the detection rate of index tumors. Biopsy core number and the location of index tumors had no effect on biochemical outcomes.

Keywords: transrectal ultrasound, TRUS, prostate biopsy, index tumor

\section{Introduction}

Prostate cancer ( $\mathrm{PCa}$ ) has become a leading cause of morbidity and mortality in men in relation with Westernization. The main diagnostic goals of clinicians are early detection, localization, staging, and prediction of aggressiveness. The identification of indolent $\mathrm{PCa}$ is important for the design of curative treatments. Assessment of prostate-specific antigen (PSA) as a marker for PCa has increased the detection rate of indolent PCa over the past two decades. ${ }^{1}$

A systematic prostate biopsy is the most common method for the diagnosis of PCa. Fink et al reviewed more than 1024 published papers with the subject of prostate biopsy $^{2}$ and found that there is no uniform procedural algorithm (indication, method, and number of cores) among urologists in European countries or in the US.

Although prostate biopsy using transrectal ultrasound (TRUS) is a common method, several reports suggested that TRUS has limitations as a screening method 
for PCa. ${ }^{3-6}$ TRUS is not reliable according to sonographers and is, therefore, not suitable for a screening test. Moreover, hypoechoic lesions (HLs) are not specific to $\mathrm{PCa},{ }^{7}$ which can be detected as isoechoic or hyperechoic lesions. ${ }^{8-10}$ Despite these limitations, TRUS is a useful tool for prostate biopsy, and TRUS-guided systematic biopsy is the gold standard method for prostate biopsy. ${ }^{11}$

Although systematic biopsy is the protocol of choice for the diagnosis of $\mathrm{PCa}$, many clinicians collect additional core biopsy specimens in suspicious cases, such as in the presence of HLs. However, there is no consensus regarding the value of additional biopsies or the number of additional cores that need to be collected.

Two large multicenter trials demonstrated that early detection is beneficial for reducing the mortality from $\mathrm{PCa}{ }^{12,13}$ Active screening for $\mathrm{PCa}$ is beneficial for reducing the mortality from $\mathrm{PCa}$, and appropriate screening is necessary for early detection.

The aim of the present study was to determine the value of HL-directed biopsy in addition to systematic biopsy for detecting $\mathrm{PCa}$ in a screening cohort of Korean men. The detection rate of index tumors in additional cores and the association of biochemical outcomes with index tumor location were investigated.

\section{Materials and methods}

This study was approved by SNUBH institutional review board (IRB) (Seoul National University Bundang Hospital: B-1412-278-111). Patient consent requirement for this study was waived by the IRB, because this was a retrospective study and only de-identified information was used; therefore, IRB has deemed it low risk.

After obtaining approval from our IRB (Seoul National University Bundang Hospital: B-1412-278-111), the records of 5037 men who underwent TRUS-guided prostate biopsy between January 2006 and August 2013 were reviewed. Patients who underwent repeat biopsies and those with insufficient data were excluded. Finally, 4144 men were included in the study. Patients were divided into Group A $(n=1006)$, which included those who underwent systematic 12-core biopsy with no cancer-suspicious lesions, and Group B $(n=485)$, which included those who underwent 13- or morecore biopsy with cancer-suspicious lesions on TRUS-guided biopsy (Figure 1).

We also analyzed cancer detection characteristics according to additional core positivity in 13 or more core biopsies. When cancer was detected in additional core, we defined it as additional core positive. When cancer was

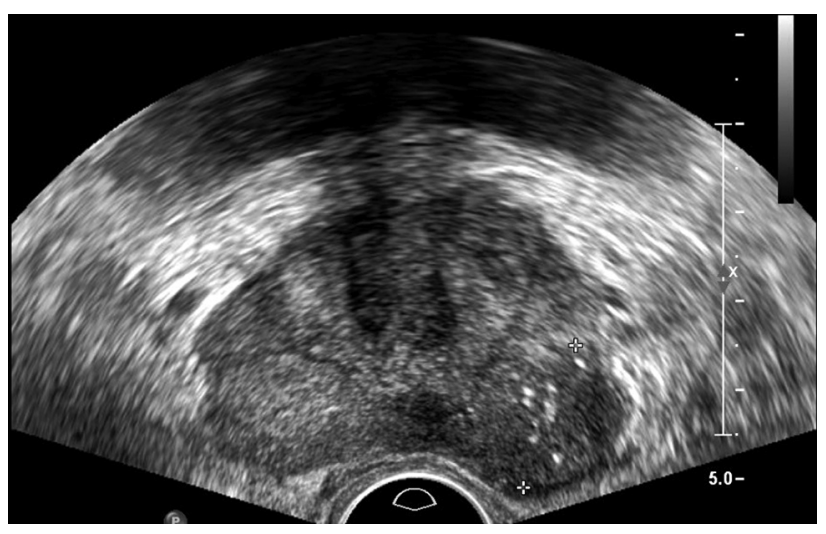

Figure I Suspicious lesion on TRUS (rim margin marked by cross). Abbreviation: TRUS, transrectal ultrasound.

not detected in additional core, we defined it as additional core negative.

In our study, patients who had index tumors in systematic cores were designated as Group I and patients who had index tumors in additional positive cores were designated as Group II.

Clinicopathologic variables were evaluated. PSA density was calculated based on the prostate volume assessed by TRUS. Cancer detection rates were calculated in each group according to index tumor location. Our pathologist defined index tumor based on size and grade. In ISUP consensus conference on the handling and staging of radical prostatectomy (RP) specimens, majority of experts voted "sized and grade" as definition of index tumor. The uropathologist in our institution also reported tertiary Gleason pattern.

We collected tumor samples from PCa tissues using TRUS-guided biopsy. After prostatectomy, we sent the specimens in a fresh status to the department of pathology. No biopsy was conducted for metastatic lesions.

For proper orientation, our pathologist measured the weight and dimensions of the entire external surface using India ink, silver nitrate, and color ink. They fixed the specimens overnight in $10 \%$ neutral formalin and clarified the glands with and without basal cell layer using mixture of CK 903 . When it was negative stains in the previous method, they usually performed P504S/AMACR immunostaining and modification of diagnosis ambiguous lesion using P63/AMACR immunostaining.

Biochemical recurrence (BCR) was evaluated according to biopsy core numbers (systematic 12-core biopsy with no cancer-suspicious lesions vs 13- or more-core biopsy with cancer-suspicious lesions) and index tumor location (index tumors in systematic 12-core biopsy vs index tumors in additional cores). BCR was defined as a PSA value $\geq 0.2 \mathrm{ng} / \mathrm{mL}$ on two consecutive measurements following RP. ${ }^{14}$ 
The Student's $t$-test and chi square test were used to analyze patient characteristics, to compare cancer detection rates between additional core negative and positive subgroups, and to analyze the detection rate of index tumor location. Logistic regression analyses were performed to analyze the significance of clinical variables. Postoperative BCR-free survival according to biopsy core number and index tumor location was calculated and compared using the Kaplan-Meier method and log-rank test. SPSS v. 22.0 (IBM ${ }^{\circledR}$ SPSS ${ }^{\circledR}$ version 22.0; IBM, Armonk, NY, USA) was used for all statistical analyses. A $p$-value $<0.05$ was considered statistically significant.

\section{Results}

Table 1 shows the baseline characteristics of patients and cancer detection rates according to biopsy core number (12 systematic cores vs 13 or more cores). Of the 4144 men included in the analysis, 3256 patients underwent 12-core systematic biopsy and 888 patients underwent 13- or morecore biopsy. A total of 1064 patients underwent RP.

Significant differences in the rate of cancer detection were observed between Group A and Group B $(p<0.001)$. Moreover, cancer detection rates according to core number differed significantly between the two groups $(p<0.001)$. Additional detailed patient characteristics are presented in Table 1.

The results of the analysis of patients who underwent 13- or more-core biopsy are presented in Table 2. Patients in the additional core positive group had more aggressive disease than those in the additional core negative group. The detection rate of index tumors was higher in additional core biopsy negative group compared with additional core positive group $(p<0.001)$. Positive core numbers and biopsy Gleason

Table I Characteristics of patients and cancer detection rates

\begin{tabular}{llll}
\hline Variables & $\begin{array}{l}\text { Group A } \\
(\mathbf{n}=\mathbf{3 2 5 6})\end{array}$ & $\begin{array}{l}\text { Group B } \\
(\mathbf{n}=\mathbf{8 8 8})\end{array}$ & p-value \\
\hline Age & $63.7 \pm 9.5$ & $66.6 \pm 8.7$ & $<0.00 \mathrm{I}$ \\
PSA, ng/mL & $10.4 \pm 40$ & $33 \pm 172.8$ & $<0.00 \mathrm{I}$ \\
Total prostate volume, cc & $44 \pm 20.9$ & $42.2 \pm 21.3$ & 0.02 \\
Transitional zone volume, cc & $21 \pm 16.7$ & $19.7 \pm 18$ & 0.042 \\
TZI & $0.44 \pm 0.16$ & $0.43 \pm 0.24$ & 0.149 \\
PSAd & $0.27 \pm 1.43$ & $0.8 \pm 4.25$ & $<0.00 \mathrm{I}$ \\
Biopsy core number & 12 & $13.4 \pm 0.8$ & $<0.00 \mathrm{I}$ \\
Cancer detection rate, n (\%) & $1006(30.9)$ & $485(54.6)$ & $<0.00 \mathrm{I}^{*}$ \\
Cancer detected core number & $\mathrm{I} . \mathrm{I} \pm 2.2$ & $3.4 \pm 4.3$ & $<0.00 \mathrm{I}$ \\
\hline
\end{tabular}

Notes: *Chi square test; Group A: I2-core systematic biopsy; Group B: I3- or more-core biopsy; TZI = transitional zone volume/total volume; PSAd = PSA prostate volume. Data are presented as mean \pm standard deviation.

Abbreviations: TZI, transitional zone index; PSAd, prostate-specific antigen density.
Table 2 Cancer detection in 13- or more-core biopsy

\begin{tabular}{llll}
\hline Variables & $\begin{array}{l}\text { Additional } \\
\text { core negative }\end{array}$ & $\begin{array}{l}\text { Additional } \\
\text { core positive }\end{array}$ & p-value \\
\hline $\begin{array}{l}\text { Number (total } \\
\mathrm{n}=485)(\%)\end{array}$ & $115(23.7)$ & $370(76.3)$ & \\
Age & $67.7 \pm 7.2$ & $68.9 \pm 7.4$ & 0.144 \\
PSA & $10.5 \pm 15.3$ & $67.9 \pm 263.7$ & $<0.001$ \\
Total prostate volume & $39.18 \pm 15.8$ & $38.8 \pm 17.2$ & 0.833 \\
Transitional zone volume & $17.8 \pm 12.9$ & $16.8 \pm 13.7$ & 0.497 \\
TZI & $0.42 \pm 0.14$ & $0.4 \pm 0.17$ & 0.216 \\
PSAd & $0.3 \pm 0.42$ & $1.64 \pm 6.49$ & $<0.001$ \\
Biopsy core numbers & $13.2 \pm 0.7$ & $13.4 \pm 0.7$ & 0.031 \\
Positive core numbers & $2.4 \pm 1.9$ & $7.5 \pm 3.7$ & $<0.001$ \\
Biopsy Gleason score & & & \\
$\leq 6$ & $78(67.8)$ & $43(11.6)$ & $<0.001^{*}$ \\
$7(3+4)$ & $19(16.5)$ & $99(26.8)$ & \\
$7(4+3)$ & $9(7.8)$ & $55(14.9)$ & \\
8 & $4(3.5)$ & $109(29.5)$ & \\
$\geq 9$ & $5(4.3)$ & $64(17.3)$ & \\
\hline
\end{tabular}

Note: $*$ Chi square test.

Abbreviations: PSA, prostate-specific antigen; TZI, transitional zone index; PSAd, prostate-specific antigen density.

scores (GS) differed significantly according to additional core positive status $(p<0.001)$. Additional detailed variables are presented in Table 2.

Regarding postoperative BCR-free survival, there were no significant differences between the groups according to biopsy core numbers (log-rank test: $p=0.583$ ) (Figure 2).

The detection rates of index tumors according to index tumor location are shown in Table 3. Differences in the distribution of variables were observed between the subgroups. The detection rate of index tumors was higher in systematic cores with additional core biopsy than in additional cores with additional core biopsy $(p<0.001)$.

Biochemical outcomes did not differ significantly according to index tumor location (log-rank test: $p=0.165$ ) (Figure 3).

In the multivariate logistic regression analysis, age, PSA, prostate volume, and biopsy core number were significant factors for PCa detection using TRUS biopsy (Table 4). Biopsy core number was the most powerful detector of $\mathrm{PCa}$ using TRUS $(p<0.001)$.

\section{Discussion}

As the biopsy core number increased, the cancer detection rate increased. The additional core positive group had a higher GS than the additional core negative group. However, additional core biopsies had no effect on the detection rate of index tumors. Moreover, additional core biopsies and the location of index tumors were not significant factors affecting biochemical outcomes. 




Figure 2 Biochemical recurrence as evaluated by Kaplan-Meier curve (BCR) according to biopsy core number (line with circles: I2-core systemic cores; line with rhombus: 13 or more cores).

Table 3 Detection rate of index tumors in additional positive cores and systematic cores

\begin{tabular}{|c|c|c|c|}
\hline Variables & Group I & Group II & $p$-value \\
\hline Number (total $n=|49|$ ) (\%) & 1258 & 233 & \\
\hline Group I with additional core & $252 / 888(28.4)$ & $233 / 888(26.2)$ & $<0.001$ \\
\hline \multicolumn{4}{|l|}{ biopsies/Group B, (\%): Group } \\
\hline \multicolumn{4}{|l|}{ II/Group B, (\%) } \\
\hline Age & $67.4 \pm 7.9$ & $69 \pm 7.2$ & 0.006 \\
\hline PSA & $22.7 \pm 100$ & $66.9 \pm 282.5$ & 0.019 \\
\hline Total prostate volume & $38.9 \pm 16.5$ & $38.8 \pm 17.6$ & 0.923 \\
\hline Biopsy core numbers & $12.25 \pm 0.6$ & $13.5 \pm 0.8$ & $<0.001$ \\
\hline Positive core numbers & $3.95 \pm 3.1$ & $7 \pm 3.9$ & $<0.001$ \\
\hline \multicolumn{4}{|l|}{ Index Gleason score } \\
\hline$\leq 6$ & $605(48.1)$ & $43(18.5)$ & $<0.00 I^{*}$ \\
\hline 7 & $426(33.9)$ & $99(42.5)$ & \\
\hline 8 & $145(\mid 1.5)$ & $57(24.5)$ & \\
\hline 9 & $73(5.8)$ & $28(12.0)$ & \\
\hline 10 & $9(0.7)$ & $6(2.6)$ & \\
\hline
\end{tabular}

Notes: *Chi square test; Group I: index tumor in systematic cores; Group II: index tumor in additional positive core group.

Abbreviation: PSA, prostate-specific antigen.

Halpern et al showed that $20 \%$ of all cancers are missed in targeted biopsies and are detected only by systematic biopsy. ${ }^{15}$ These authors compared targeted biopsies with sextant core biopsies using contrast ultrasound images. In the present study, we compared additional suspicious lesion biopsies with 12-core systematic biopsies using conventional ultrasound and found that additional suspicious lesion biopsies increased the cancer detection rate by $23.7 \%$ over 12 -core systematic biopsies.

A tendency to implement biopsies with additional cores to improve cancer detection rate was suggested in previous studies. ${ }^{16-19}$ Many series reported on comparisons with sextant biopsies. However, there is no consensus regarding the number of additional cores. Gosselaar et al did not reach a conclusion regarding whether PCa detection is improved by collecting additional cores from a suspicious lesion on TRUS in extended biopsy schemes that included 14 cores in men with no previous biopsy results. ${ }^{20}$ However, we demonstrated the utility of additional core biopsies in patients with suspicious lesions on TRUS. With considering age, PSA level, and PSA density, cancer-detected core number differed according to each group. Average added core number just 1.4 in each group, however difference of cancer detected core number was over 2 cores. Moreover, we found that additional core biopsies were not associated with the detection rate of index tumors. 


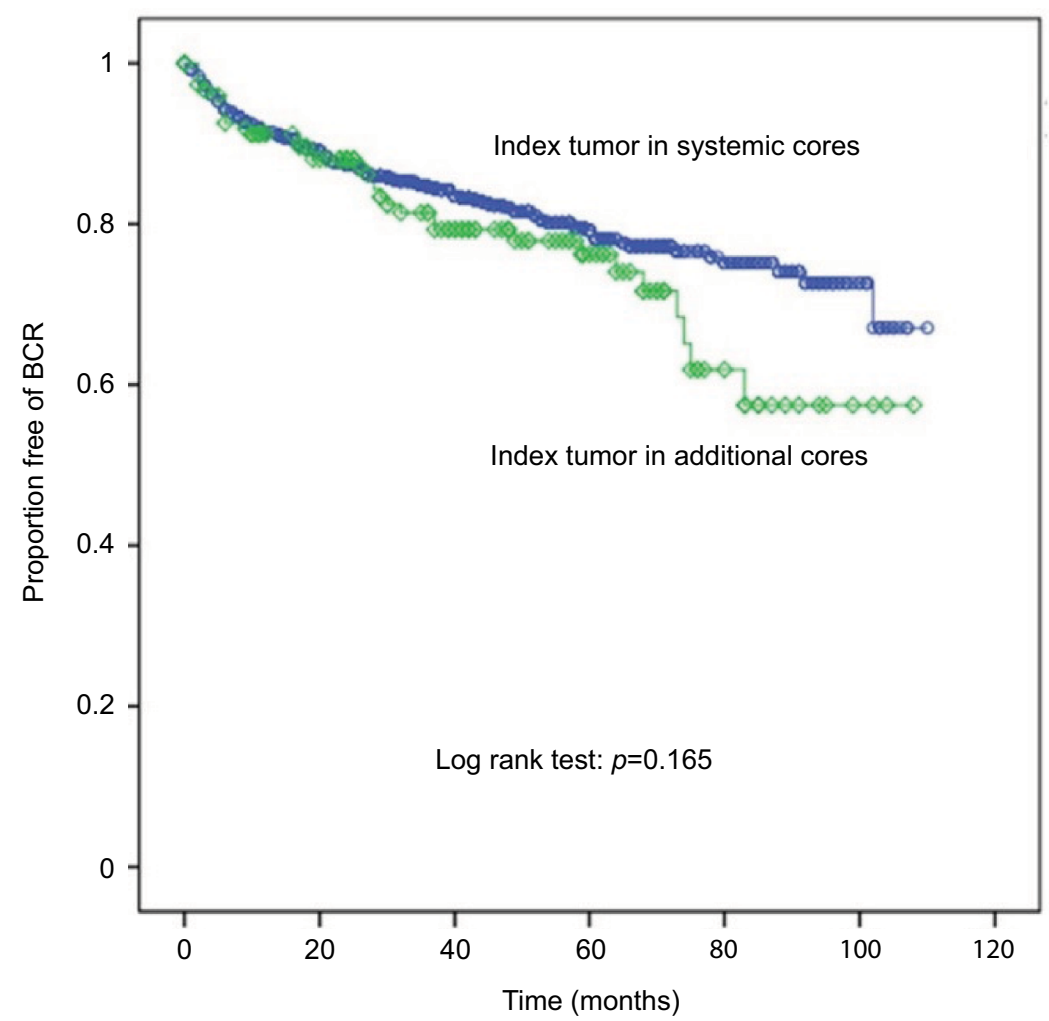

Figure 3 Biochemical recurrence as evaluated by Kaplan-Meier curve (BCR) according to index tumor location (line with circle: index tumor in systemic cores; line with rhombus: index tumor in additional cores).

Table 4 Logistic regression analysis for the identification of significant factors during TRUS biopsy

\begin{tabular}{llll}
\hline Variables & OR & $\mathbf{9 5 \%} \mathbf{C l}$ & $\boldsymbol{p}$-value \\
\hline Age $(\leq 65$ vs $>65)$ & $2.21 \mathrm{I}$ & $1.93 \mathrm{I}-2.533$ & $<0.00 \mathrm{I}$ \\
PSA $(\leq 4$ vs $>4)$ & 2.173 & $1.823-2.589$ & $<0.00 \mathrm{I}$ \\
Total prostate volume $(\leq 20$ vs $>20)$ & 0.5 & $0.347-0.722$ & $<0.00$ I \\
Biopsy core number $(\leq 12$ vs $>12)$ & 2.6 & $2.22 \mathrm{I}-3.043$ & $<0.00 \mathrm{I}$ \\
\hline
\end{tabular}

Abbreviations: TRUS, TRUS, transrectal ultrasound; OR, odd ratio; $\mathrm{Cl}$, confidence interval; PSA, prostate-specific antigen.

Flanigan et al reported on the limited accuracy of TRUS for the identification and localization of $\mathrm{PCa}^{21}{ }^{21}$ th that study, of the 6630 subjects who were screened for PCa, 251 were diagnosed with PCa. Of these, 131 (52\%) would have been missed if only the exact site of the suspicious lesion had been biopsied. Other previous studies reported that the sensitivity of TRUS for the detection of $\mathrm{PCa}$ is $52 \%$ and $53 \%$, while the specificity is $68 \%$ and $75 \%{ }^{22,3}$ Multi-parameter magnetic resonance imaging (MRI) including apparent diffusion coefficient (ADC) is emerging as a helpful tool for the detection of indolent tumors. ${ }^{23,24}$ Although MRI can increase the accuracy of detection, it is a costly procedure and is, therefore, not feasible for routine evaluations. MRI can be used as a staging work-up and for the selection of active surveillance candidates or as a follow-up tool. Therefore, we focused on the utility of TRUS, which had the advantage of cost-effectiveness.

Onur et al analyzed HL-directed biopsies on a per core basis and reported that a biopsy from a suspicious lesion had similar sensitivity as that from an isoechoic region. ${ }^{25}$ Although the echogenicity pattern of TRUS included impalpable lesions, the results suggest the use of a randomized biopsy strategy. Previous studies demonstrated that systematic biopsy should be chosen instead of lesion-directed cores. ${ }^{26,27}$ Because there is a low positive biopsy rate for hypoechoic areas and a considerable chance of cancer detected in isoechoic areas. Scattoni et al reported a detection rate of $\mathrm{PCa}$ of $38.7 \%$ for 10 -core biopsy and $42.2 \%$ for 14 -core biopsy. ${ }^{28}$ This represented an increase of $3.5 \%$ in the overall detection rate of $\mathrm{PCa}$ by analysis of four additional cores.

By contrast, Norberg et al reported that additional targeted biopsies from suspicious lesions on TRUS increased the relative sensitivity from $85 \%$ to $93 \%$ in a prospective trial of $>500$ men with suspicious lesions. ${ }^{29}$

This is consistent with the results of the present study, which demonstrated the utility of additional biopsies of suspicious lesions. Compared with systematic biopsies, additional core biopsies from suspicious lesions resulted 
in higher detection rates and higher GS compared with the suspicious negative group.

The present results also showed that biopsy core number and the location of index tumors did not affect the biochemical outcomes.

The present study had several limitations. First, there were limitations associated with the retrospective nature of the study. Second, the analysis included only TRUS images without multi-parameter MRI, which is valuable for the detection of PCa. However, we were unable to include multiparameter MRI results in our analysis. Third, TRUS was not performed by one sonographer, but rather by two different uroradiologic specialists. Despite these limitations, clinically meaningful values were obtained using TRUS, which has the advantage of cost-effectiveness and is a commonly used tool. According to our results, we suggest that clinicians need to add two or more additional biopsies based on patient's age, PSA level, prostate volume, and suspicious lesion. Further studies are necessary to address an optimal number beyond systematic biopsy based on complications.

\section{Conclusion}

Additional core biopsies can increase cancer detection rate, although they do not increase the detection rate of index tumors. Biopsy core number and the location of index tumors had no effect on the biochemical outcomes. However, additional core biopsies were associated with higher GS PCa. Detection of suspicious lesions on TRUS should be analyzed by additional biopsies to increase the detection rate while considering that additional biopsy cores do not affect the biochemical outcome.

\section{Disclosure}

The authors report no conflicts of interest in this work.

\section{References}

1. Sarma AV, Schottenfeld D. Prostate cancer incidence, mortality, and survival trends in the United States: 1981-2001. Semin Urol Oncol. 2002;20(1):3-9.

2. Fink KG, Schmid HP, Paras L, Schmeller NT. Prostate biopsy in Central Europe: results of a survey of indication, patient preparation and biopsy technique. Urol Int. 2007;79(1):60-66.

3. Terris MK, Freiha FS, McNeal JE, Stamey TA. Efficacy of transrectal ultrasound for identification of clinically undetected prostate cancer. $J$ Urol. 1991;146(1):78-83.

4. Rietbergen JB, Kranse R, Kirkels WJ, De Koning HJ, Schroder FH. Evaluation of prostate-specific antigen, digital rectal examination and transrectal ultrasonography in population-based screening for prostate cancer: improving the efficiency of early detection. Br J Urol. 1997;79 (Suppl 2):57-63.
5. Kranse R, Beemsterboer P, Rietbergen J, et al. Predictors for biopsy outcome in the European Randomized Study of Screening for Prostate Cancer (Rotterdam region). Prostate. 1999;39(4):316-322.

6. Beemsterboer PM, Kranse R, de Koning HJ, Habbema JD, Schroder FH. Changing role of 3 screening modalities in the European randomized study of screening for prostate cancer (Rotterdam). Int J Cancer. 1999;84(4):437-441.

7. Littrup PJ, Bailey SE. Prostate cancer: the role of transrectal ultrasound and its impact on cancer detection and management. Radiol Clin North Am. 2000;38(1):87-113.

8. Heijmink SW, van Moerkerk H, Kiemeney LA, et al. A comparison of the diagnostic performance of systematic versus ultrasound-guided biopsies of prostate cancer. Eur Radiol. 2006;16(4):927-938.

9. Vo T, Rifkin MD, Peters TL. Should ultrasound criteria of the prostate be redefined to better evaluate when and where to biopsy. Ultrasound Q. 2001;17(3):171-176.

10. Shinohara K, Wheeler TM, Scardino PT. The appearance of prostate cancer on transrectal ultrasonography: correlation of imaging and pathological examinations. J Urol. 1989;142(1):76-82.

11. Durkan GC, Greene DR. Diagnostic dilemmas in detection of prostate cancer in patients undergoing transrectal ultrasound-guided needle biopsy of the prostate. Prostate Cancer Prostatic Dis. 2000;3(1):13-20.

12. Roobol MJ, Schroder FH. The European Randomized Study of Screening for Prostate Cancer (ERSPC): rationale, structure and preliminary results 1994-2003. BJU Int. 2003;92 (Suppl 2):1-124.

13. Prorok PC, Andriole GL, Bresalier RS, et al. Design of the Prostate, Lung,Colorectal and Ovarian (PLCO) Cancer Screening Trial. Control Clin Trials. 2000;21 (Suppl 6):273S-309S.

14. Abdollah F, Karnes RJ, Suardi N, et al. Predicting survival of patients with node-positive prostate cancer following multimodal treatment. Eur Urol. 2014;65(3):554-562.

15. Halpern EJ, Ramey JR, Strup SE, et al. Detection of prostate carcinoma with contrast-enhanced sonography using intermittent harmonic imaging. Cancer. 2005;104(11):2373-2383.

16. Presti JC Jr, O’Dowd GJ, Miller MC, Mattu R, Veltri RW. Extended peripheral zone biopsy schemes increase cancer detection rates and minimize variance in prostate specific antigen and age related cancer rates: results of a community multi-practice study. J Urol. 2003;169(1):125-129.

17. Stewart CS, Leibovich BC, Weaver AL, Lieber MM. Prostate cancer diagnosis using a saturation needle biopsy technique after previous negative sextant biopsies. J Urol. 2001;166(1):86-91.

18. Levine MA, Ittman M, Melamed J, Lepor H. Two consecutive sets of transrectal ultrasound guided sextant biopsies of the prostate for the detection of prostate cancer. J Urol. 1998;159(2):471-475.

19. Vashi AR, Wojno KJ, Gillespie B, Oesterling JE. A model for the number of cores per prostate biopsy based on patient age and prostate gland. $J$ Urol. 1998;159(3):920-924.

20. Gosselaar C, Roobol MJ, Roemeling S, et al. The value of an additional hypoechoic lesion-directed biopsy core for detecting prostate cancer. BJU Int. 2008;101(6):685-690.

21. Flanigan RC, Catalona WJ, Richie JP, et al. Accuracy of digital rectal examination and transrectal ultrasonography in localizing prostate cancer. J Urol. 1994;152(5 Pt 1):1506-1509.

22. Carter HB, Hamper UM, Sheth S, et al. Evaluation of transrectal ultrasound in the early detection of prostate cancer. J Urol. 1989;142(4): 1008-1010.

23. Mullins JK, Bonekamp D, Landis P, et al. Multiparametric magnetic resonance imaging findings in men with low-risk prostate cancer followed using active surveillance. BJU Int. 2013;111(7):1037-1045.

24. Park BH, Jeon HG, Choo SH, et al. Role of multiparametric 3.0-Tesla magnetic resonance imaging in patients with prostate cancer eligible for active surveillance. BJU Int. 2014;113(6):864-870.

25. Onur R, Littrup PJ, Pontes JE, Bianco FJ Jr. Contemporary impact of transrectal ultrasound lesions for prostate cancer detection. J Urol. 2004;172(2):512-514. 
26. Hammerer P, Huland H. Systematic sextant biopsies in 651 patients referred for prostate evaluation. J Urol. 1994;151(1):99-102.

27. Vallancien G, Prapotnich D, Veillon B, Brisset JM, Andre-Bougaran J. Systematic prostatic biopsies in 100 men with no suspicion of cancer on digital rectal examination. $J$ Urol. 1991;146(5):1308-1312.
28. Scattoni V, Maccagnano C, Zanni G, et al. Is extended and saturation biopsy necessary? Int J Urol. 2010;17(5):432-447.

29. Norberg M, Egevad L, Holmberg L, et al. The sextant protocol for ultrasound-guided core biopsies of the prostate underestimates the presence of cancer. Urology. 1997;50(4):562-566.
Cancer Management and Research

\section{Publish your work in this journal}

Cancer Management and Research is an international, peer-reviewed open access journal focusing on cancer research and the optimal use of preventative and integrated treatment interventions to achieve improved outcomes, enhanced survival and quality of life for the cancer patient. The manuscript management system is completely online and includes a very quick and fair peer-review system, which is all easy to use. Visit $\mathrm{http}: / / \mathrm{www}$.dovepress.com/testimonials.php to read real quotes from published authors. 